\title{
Jet Measurements at STAR
}

\author{
Jan Rusnak*i \\ Nuclear Physics Institute, Czech Academy of Sciences, \\ Na Truhlarce 39/64, 18086 Prague, Czech Republic \\ E-mail: rusn@email.cz
}

\begin{abstract}
Jets are an important tool to explore the properties of the hot and dense nuclear matter created in heavy-ion collisions. However, full jet reconstruction in such events is a challenging task due to the extremely large and fluctuating background, which generates a large population of combinatorial jets that overwhelm the true hard jet population.

In order to carry out accurate, data-driven jet measurements over a broad kinematic range in these conditions of small signal to background, we use modern approaches in order to measure inclusive charged jet distributions and semi-inclusive charged jet distributions recoiling from a high momentum trigger in central $\mathrm{Au}+\mathrm{Au}$ collisions. In addition we present the measurement of the di-jet transverse momentum asymmetry, $A_{\mathrm{J}}$. These jet measurements allow a direct comparison of jet quenching at RHIC and the LHC.
\end{abstract}

7th International Conference on Physics and Astrophysics of Quark Gluon Plasma

1-5 February, 2015

Kolkata, India

\footnotetext{
${ }^{*}$ Speaker.

${ }^{\dagger}$ For the STAR collaboration.
} 


\section{Motivation}

Hard scattering processes produce partons with high transverse momentum $p_{\mathrm{T}}$. These recoiled partons fragment and then hadronize into a collimated stream of mesons and baryons. These final state hadrons are clustered into a jet, which should reflect the kinematics of the hard scattered parton. Due to the fact that jets originate from hard processes, perturbative QCD is applicable for calculation of their cross-section. The pQCD calculations describe measurements of jet production in elementary collisions (pp, e+e) with high accuracy [1].

In heavy-ion collisions, jets are produced in an early stage of the collision, allowing them to interact with the surrounding medium (quark gluon plasma, QGP). A parton traverses through the hot, dense, strongly interacting medium, losing energy via radiative and collisional processes, which will soften and broaden the final state jet [2]. By comparing properties of jets in heavy-ion collisions with theoretically well described jets in elementary collisions, one can indirectly study properties of the QGP. Jets are therefore a good probe of the QCD matter.

\section{Experimental Setup and Data-sets}

Analyses presented in this proceeding are based on data recorded by the Solenoidal Tracker At RHIC (STAR), which is a complex detector consisting of several sub-detectors and full azimuthal coverage. Its main sub-detector is a large Time Projection Chamber (TPC) capable of tracking and identification of charged particles down to a transverse momentum of $100 \mathrm{MeV} / c$. The TPC is surrounded by a Barrel Electromagnetic Calorimeter (BEMC) and other detectors such as Time Of Flight (TOF) and Muon Telescope Detector (MTD) and a massive solenoidal 0.5 T magnet. Full description of the STAR experiment and its main sub-detectors can be found in $[3,4,5]$.

Inclusive and semi-inclusive jet analyses use minimum bias (MB) data from $\mathrm{Au}+\mathrm{Au}$ collisions at an energy per nucleon pair of $\sqrt{s_{N N}}=200 \mathrm{GeV}$ recorded by the STAR experiment at RHIC in 2011. Events with TPC multiplicities corresponding to $0-10 \%$ and $60-80 \%$ most central $\mathrm{Au}+\mathrm{Au}$ collisions and having the $z$ position of the primary vertex within $30 \mathrm{~cm}$ from the center of the TPC are further used in these data analyses.

The $\left|A_{\mathrm{J}}\right|$ analysis uses High-Tower (HT) triggered events (events with energy deposit in a BEMC tower above a certain threshold) recorded by the STAR experiment in $\mathrm{Au}+\mathrm{Au}$ collisions at $\sqrt{s_{N N}}=200 \mathrm{GeV}$ in 2007 with the TPC multiplicity corresponding to the $0-20 \%$ most central $\mathrm{Au}+\mathrm{Au}$ collisions.

\section{Jet Reconstruction}

Inclusive and semi-inclusive jet analyses presented below use the TPC tracks to reconstruct charged jets. The TPC tracks were required to have transverse momenta $p_{\mathrm{T}}>0.2 \mathrm{GeV} / c$, pseudorapidity $|\eta|<1$ and at least 20 TPC space-points.

The di-jet asymmetry $\left|A_{\mathrm{J}}\right|$ measurement is based on full jets, utilizing also energy deposited in the BEMC towers. $100 \%$ hadronic correction was applied to the BEMC towers (the entire $p_{\mathrm{T}}$ of a track matched to a calorimeter tower is subtracted from that tower's energy). 
The TPC tracks (and calorimetric towers) were collected into jets using the anti- $\mathrm{k}_{\mathrm{T}}$ algorithm provided by the FastJet package [6,7]. The jet area $A$ was then calculated using the population of soft "ghost particles" [8]. The background energy was subtracted jet-by-jet from the reconstructed jet transverse momentum by

$$
p_{\mathrm{T}, \mathrm{jet}}^{\mathrm{reco}}=p_{\mathrm{T}, \mathrm{jet}}-\rho \times A
$$

where $\rho$ is the background median energy density calculated for each event as

$$
\rho=\operatorname{med}\left\{\frac{p_{\mathrm{T}, \mathrm{jet}}^{i}}{A_{i}}\right\}
$$

where the index $i$ goes through all reconstructed jets in the event (with exclusion of the 2-3 hardest jets in the event). The background jets are reconstructed using the $\mathrm{k}_{\mathrm{T}}$ algorithm.

The jet resolution parameter $R$, which determines the size of the reconstructed jet, was chosen to be $0.2,0.3$ and 0.4 . All jets are required to lie within the fiducial rapidity acceptance $|\eta|<1-R$.

\section{Inclusive Charged Jet Measurements}

The inclusive jet cross-section in $\mathrm{p}+\mathrm{p}$ collisions represents an important observable that is calculable in pQCD. In heavy-ion collisions jet fragmentation will be affected by the presence of the hot and dense medium created in these collisions. Therefore it is of a high importance to compare the $p+p$ measurements with those in the nucleus-nucleus collisions.

Jet reconstruction in the environment of the heavy-ion collisions has to correct for the large and highly fluctuating background. In order to reduce the combinatorial jet background in the $\mathrm{Au}+\mathrm{Au}$ collisions a cut on the transverse momentum of the leading hadron in the jet (hadron with the highest transverse momentum) is applied. In our case we impose a cut of $p_{\mathrm{T}}^{\text {leading }}>5 \mathrm{GeV} / \mathrm{c}$. Such a cut induces a fragmentation bias, which needs to be systematically studied (see Fig. 1). However, as there are no requirements on the rest of the jet constituents, the effect of the bias is negligible for high-momentum jets.

The most significant effect which needs to be taken into account in this analysis is the large background fluctuation. The jet response to such fluctuations is measured on ensemble basis by embedding simulated jets into real events. By comparing the momenta of the embedded jet and the geometrically matched reconstructed jet one can evaluate the response in terms of the $\delta p_{\mathrm{T}}$ distribution

$$
\delta p_{\mathrm{T}}=p_{\mathrm{T}, \mathrm{jet}}^{\mathrm{reco}}-p_{\mathrm{T}, \mathrm{jet}}^{\mathrm{emb}}=p_{\mathrm{T}, \mathrm{jet}}-\rho \times A-p_{\mathrm{T}, \mathrm{jet}}^{\mathrm{emb}}
$$

It has been shown [9] that the shape of the $\delta p_{\mathrm{T}}$ distribution is almost independent of the fragmentation model of the embedded jet. Also the dependency of the $\delta p_{\mathrm{T}}$ distribution on the momentum of the embedded probe is very weak as can be seen in Fig. 2.

The $\delta p_{\mathrm{T}}$ distribution is sampled in order to produce the corresponding response matrix $\mathbf{R}_{\delta p_{\mathrm{T}}}\left[p_{\mathrm{T}, \mathrm{jet}}^{\mathrm{reco}}, p_{\mathrm{T}, \mathrm{jet}}^{\mathrm{emb}}\right]=\mathbf{R}_{\delta p_{\mathrm{T}}}\left[p_{\mathrm{T}, \mathrm{jet}}^{\mathrm{emb}}+\delta p_{\mathrm{T}}, p_{\mathrm{T}, \mathrm{jet}}^{\mathrm{emb}}\right]$. The detector response is approximated by the parametrization of the TPC tracking efficiency which was estimated by the embedding of simulated tracks into real events and running the TPC track reconstruction algorithm on such events. 


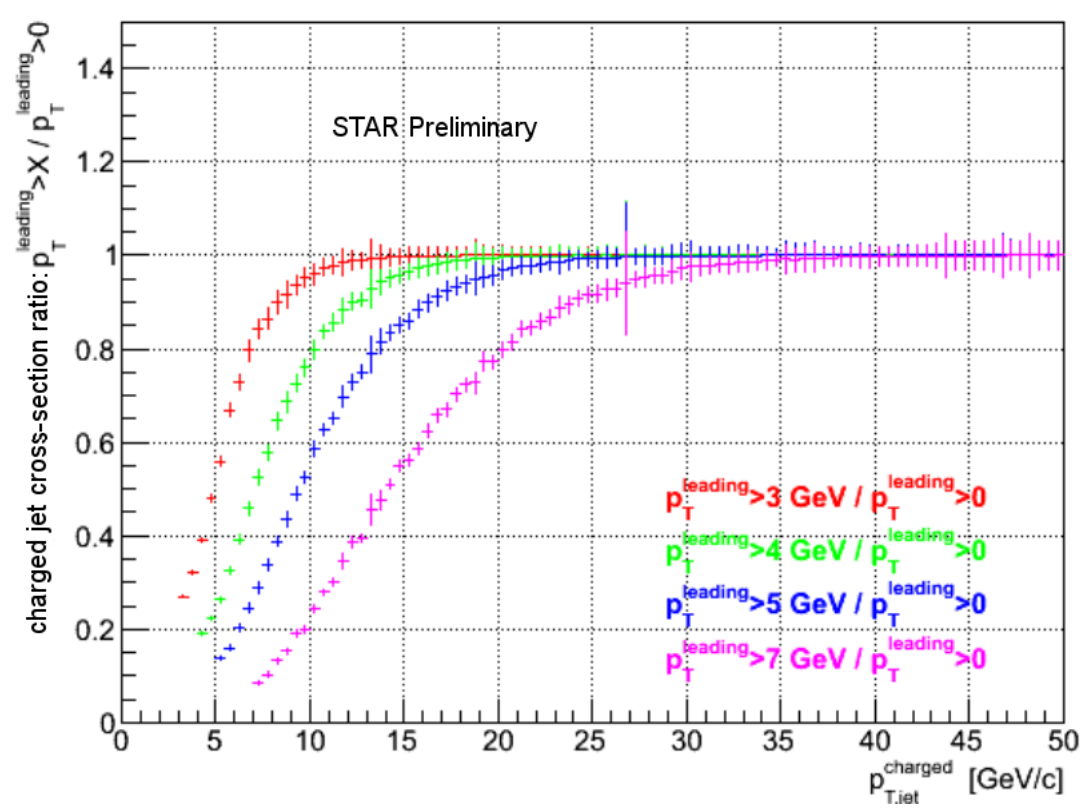

Figure 1: Effect of the $p_{\mathrm{T}}^{\text {leading }}$ cut on PYTHIA charged jet spectra at $\sqrt{s_{N N}}=200 \mathrm{GeV}$ and within STAR acceptance.

The tracking efficiency for hadrons is $68 \%$ at $p_{\mathrm{T}}=0.5 \mathrm{GeV} / c$ and $72 \%$ for $p_{\mathrm{T}}>1 \mathrm{GeV} / c$ in central collisions.

Using jets generated by PYTHIA, a detector response matrix $\mathbf{R}_{\text {instr }}\left[p_{\mathrm{T}}^{\mathrm{det}}, p_{\mathrm{T}}^{\text {part }}\right]$ was calculated, where $p_{\mathrm{T}}^{\text {part }}$ is the transverse momentum of the generated (and reconstructed) PYTHIA jet and $p_{\mathrm{T}}^{\text {det }}$ is the momentum of the same (geometrically matched) jet after the tracking efficiency was applied to its constituents.

The full correction response matrix $\mathbf{R}_{\text {full }}$ is obtained by multiplying the two response matrices

$$
\mathbf{R}_{f u l l}=\mathbf{R}_{\delta p_{\mathrm{T}}} \times \mathbf{R}_{\text {instr }}
$$

Using this response matrix and a power-law prior distribution, the jet transverse momentum spectrum is corrected for the background fluctuations and the TPC tracking efficiency via an iterative Bayesian unfolding [10]. No $p_{\mathrm{T}}$ cut-off is applied on the jet spectrum prior unfolding.

The systematic uncertainty arising from the unfolding was estimated by varying the power of the initial power-law prior distribution (from -3 to -6 ) and by varying the number of iterations. Another component of the systematic uncertainty comes from the uncertainty on the tracking efficiency. It was estimated by varying the tracking efficiency $\pm 5 \%$ (absolute).

After the unfolding procedure the jet spectrum is further corrected for the jet reconstruction efficiency by multiplying a given momentum bin with the inverse value of the corresponding efficiency. When calculating the jet reconstruction efficiency, one should not compare directly detector-level and particle-level distributions bin-by-bin. Due to the detector effects, a jet having $p_{\mathrm{T}, \mathrm{jet}}^{\text {part }}$ will be typically detected with $p_{\mathrm{T}, \mathrm{jet}}^{\mathrm{det}} \neq p_{\mathrm{T}, \mathrm{jet}}^{\mathrm{part}}$ and thus potentially migrate from $i$-th $p_{\mathrm{T}}$ bin to $j$-th. Comparing $i$-th bin of particle-level spectrum with $i$-th bin of detector level spectrum is 


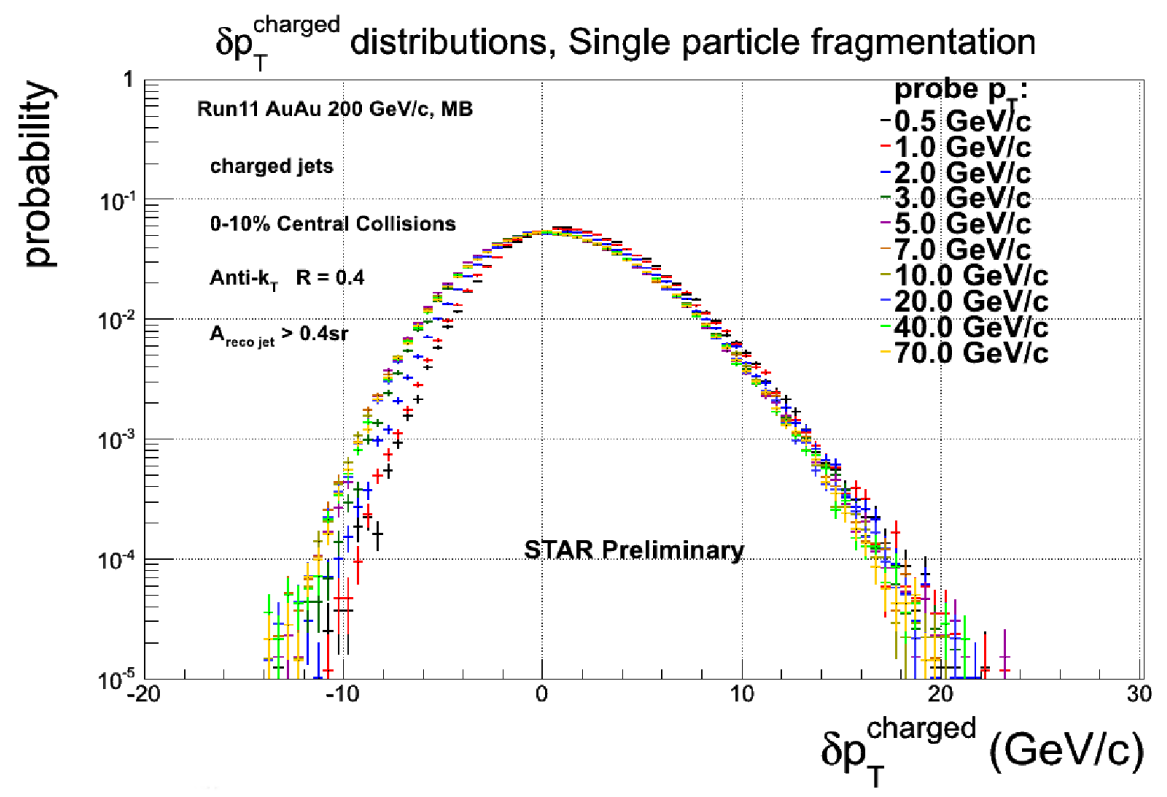

Figure 2: $\delta p_{\mathrm{T}}$ distribution for central $\mathrm{Au}+\mathrm{Au}$ collisions at $\sqrt{s_{N N}}=200 \mathrm{GeV}$ for several momenta of embedded probes.

therefore not a good strategy and one has to take into account the above mentioned bin migration. Jet reconstruction efficiency was thus defined with respect to the particle-level jets as

$$
\varepsilon\left(p_{\mathrm{T}, \mathrm{jet}}^{\text {part }}\right)=\frac{\frac{\mathrm{dN}_{\mathrm{jet}}}{\mathrm{d} p_{\mathrm{T}, \mathrm{jet}}^{\text {det }}} \otimes \widetilde{\mathbf{R}_{\text {instr }}^{-1}}\left[p_{\mathrm{T}, \mathrm{jet}}^{\mathrm{part}} \rightarrow p_{\mathrm{T}, \mathrm{jet}}^{\mathrm{det}}\right]}{\frac{\mathrm{d} \mathbf{N}_{\text {jet }}}{\mathrm{d} p_{\mathrm{T}, \mathrm{jet}}^{\text {patt }}}}
$$

where $\frac{\mathrm{dN} \mathrm{jet}_{\mathrm{jet}}}{\mathrm{d} p_{\mathrm{T}, \mathrm{t} \text { et }}}$ and $\frac{\mathrm{dN} \mathrm{N}_{\mathrm{jet}}}{\mathrm{d} p_{\mathrm{T}, \mathrm{jet}}^{\text {pat }}}$ are the detector-level and particle-level jet spectra. The expression in the numerator of Equation (4.3) is to be understood as unfolded and regularized detector-level spectrum. Unfolding of the detector-level spectrum was done using only the instrumental response matrix $\mathbf{R}_{\text {instr. }}$.

Fig. 3 shows the inclusive charged jet spectra for jet resolution parameters $R=0.2$ and 0.3 , corrected for the background fluctuations, TPC tracking efficiency and jet reconstruction efficiency. Systematic uncertainties from unfolding and tracking efficiencies are added linearly.

The low momentum part of the spectra is biased by the cut on the momentum of the leading hadron $p_{\mathrm{T}}^{\text {leading }}>5 \mathrm{GeV} / c$. PYTHIA simulations shown on Fig. 1 reveal that the effect of this bias is significant up to momenta $p_{\mathrm{T}, \mathrm{jet}} \simeq 4 \cdot p_{\mathrm{T}}^{\text {leading }}=20 \mathrm{GeV} / c$.

In order to calculate the charged jet nuclear modification factor $R_{A A}$ a valid pp baseline is needed.

\section{Semi-Inclusive Recoil Charged Jet Measurements}

A way to avoid the bias on the jet fragmentation caused by imposing a momentum cut on the jet constituents is to bias the event selection instead. This can be done by selecting a high momentum 

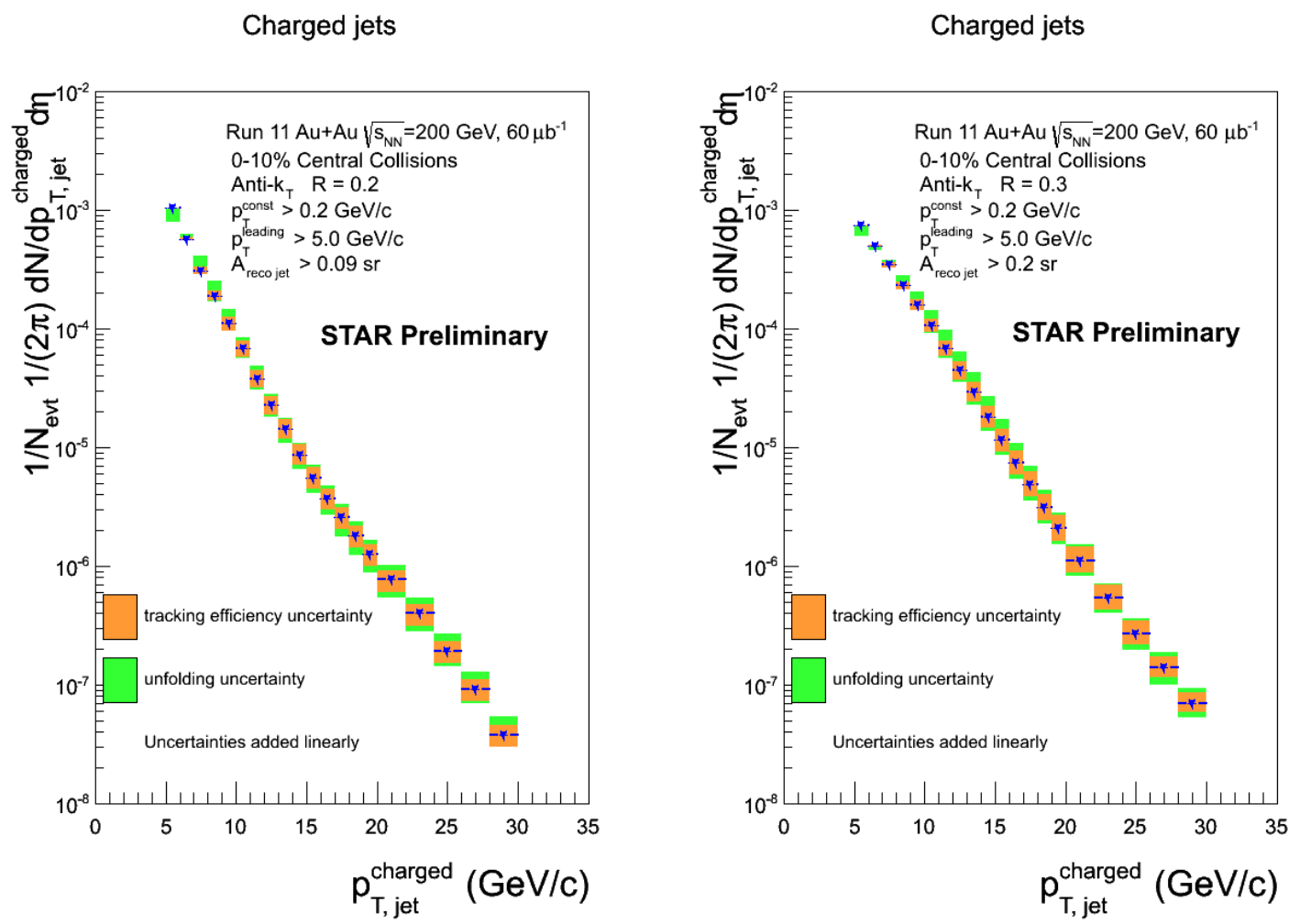

Figure 3: The unfolded $p_{\mathrm{T}}$ spectrum of charged jets in central $\mathrm{Au}+\mathrm{Au}$ collisions at $\sqrt{s_{N N}}=200 \mathrm{GeV}$ for $R$ $=0.2$ (left) and $R=0.3$ (right).

hadron trigger. Jets reconstructed on the away side in azimuth will be completely unbiased with respect to their fragmentation (there is no need to impose a cut on the constituents' momenta). Furthermore such a jet population will be biased towards larger path-length in the medium making it a viable probe of the medium. An observable which is easily calculable by means of pQCD is the recoil jet yield per trigger. Jets are reconstructed with anti- $\mathrm{k}_{\mathrm{T}}$ algorithm with resolution parameter $R=0.3$. The azimuthal acceptance of the recoil jets is chosen to be within 45 degrees from the recoil direction opposite to the trigger hadron,

$$
\left|\phi_{\text {Jet }}-\left(\phi_{\text {Trig }}+\pi\right)\right| \leq \pi / 4 .
$$

The hadron trigger momentum range is chosen to be $9-19 \mathrm{GeV} / c$.

A unique mixed event method was developed which should describe the background as closely as possible. The mixed event is created by replacing all tracks in a real event by randomly selected tracks from randomly chosen events (one track from one event) from the same centrality bin, event plane direction bin and primary vertex $z$ position bin. All high momentum tracks $\left(p_{\mathrm{T}}>3 \mathrm{GeV} / c\right)$ which could possibly come from a hard jet are discarded. This creates a perfectly uncorrelated sample of tracks which preserve some important features of the real events, e.g. the detector acceptance inefficiencies.

The jet reconstruction algorithm is then run on the mixed event (ME) sample with the same settings as on the real events ("same event", SE). The obtained ME jet spectrum is normalized by 

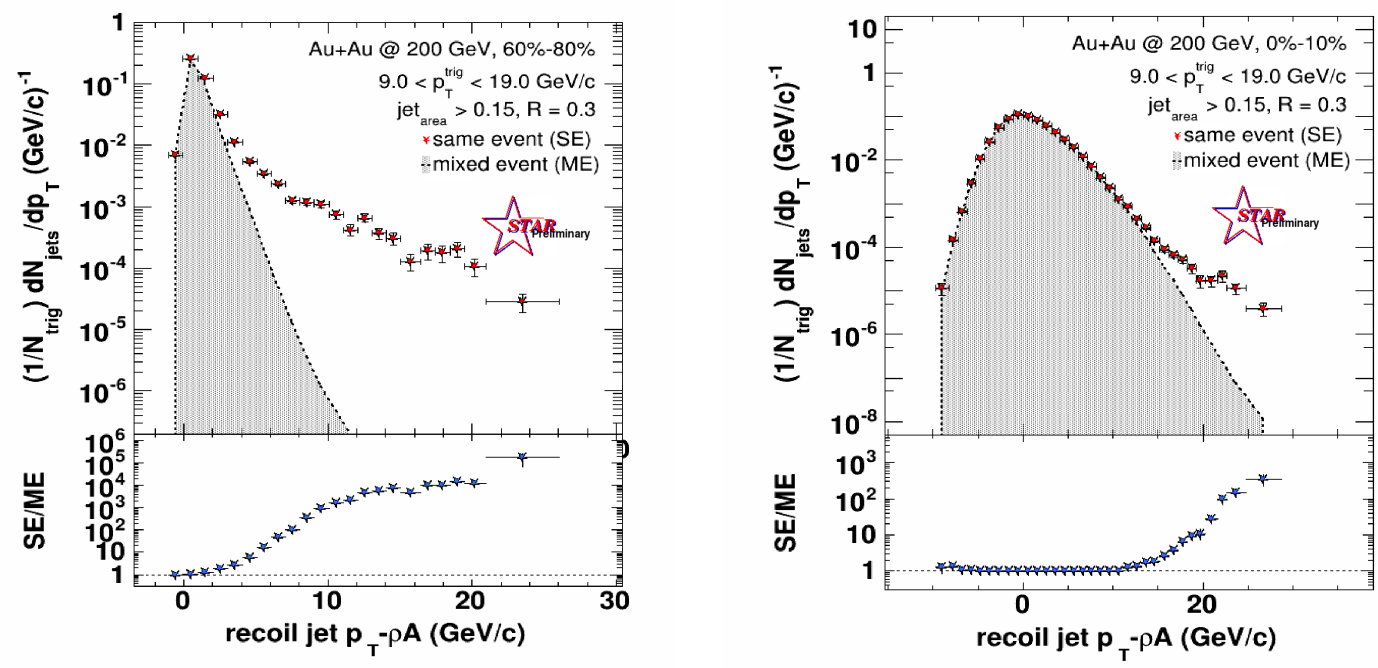

Figure 4: Uncorrected recoil charged jet spectrum in comparison with mixed event spectrum in peripheral (left) and central (right) $\mathrm{Au}+\mathrm{Au}$ collisions at $\sqrt{s_{N N}}=200 \mathrm{GeV}$.

matching the integral of the left hand side (LHS) of the spectrum with the LHS of the SE spectrum. The size of this normalization region was systematically varied and the resulting variance was included in the systematic uncertainties.

Fig. 4 shows both the same event and mixed event recoil charged jet spectra in peripheral and central $\mathrm{Au}+\mathrm{Au}$ collisions normalized per trigger yield. As one would expect, the background is much less severe in the peripheral collisions than in the most central ones. The bottom panels show the ratio SE/ME. In central collisions, the low momentum spectrum is dominated by the background. Since the ratio SE/ME is consistent with unity up to $10 \mathrm{GeV} / c$ in the central collisions (right bottom panel), this means the background is perfectly described by the mixed event. The recoil jet signal is then extracted by subtracting the mixed event spectrum from the same event.

The recoil jet spectrum shown in Fig. 5 is not corrected for the background fluctuations and detector effects yet. However, as a first approximation, it can be compared with PYTHIA $8 \mathrm{p}+\mathrm{p}$ spectrum smeared with $\delta p_{\mathrm{T}}$ distribution, hence simulating the effect of the presence of heavy-ion collision background, and with tracking efficiency applied to the PYTHIA jets constituents.

Such "detector level" recoil jet spectra normalized per trigger yield are shown on Fig. 5 both for the peripheral and central $\mathrm{Au}+\mathrm{Au}$ collisions. In peripheral collisions there is no sign of a suppression of the $\mathrm{Au}+\mathrm{Au}$ spectra with respect to PYTHIA, as can be seen from the ratio plot in the bottom. However in central $\mathrm{Au}+\mathrm{Au}$ collisions a strong suppression is evident.

\section{6. $\left|A_{\mathrm{J}}\right|$ Measurements}

The di-jet imbalance $A_{\mathrm{J}}$ is defined as

$$
A_{\mathrm{J}}=\frac{p_{\mathrm{T}, 1}-p_{\mathrm{T}, 2}}{p_{\mathrm{T}, 1}+p_{\mathrm{T}, 2}}
$$



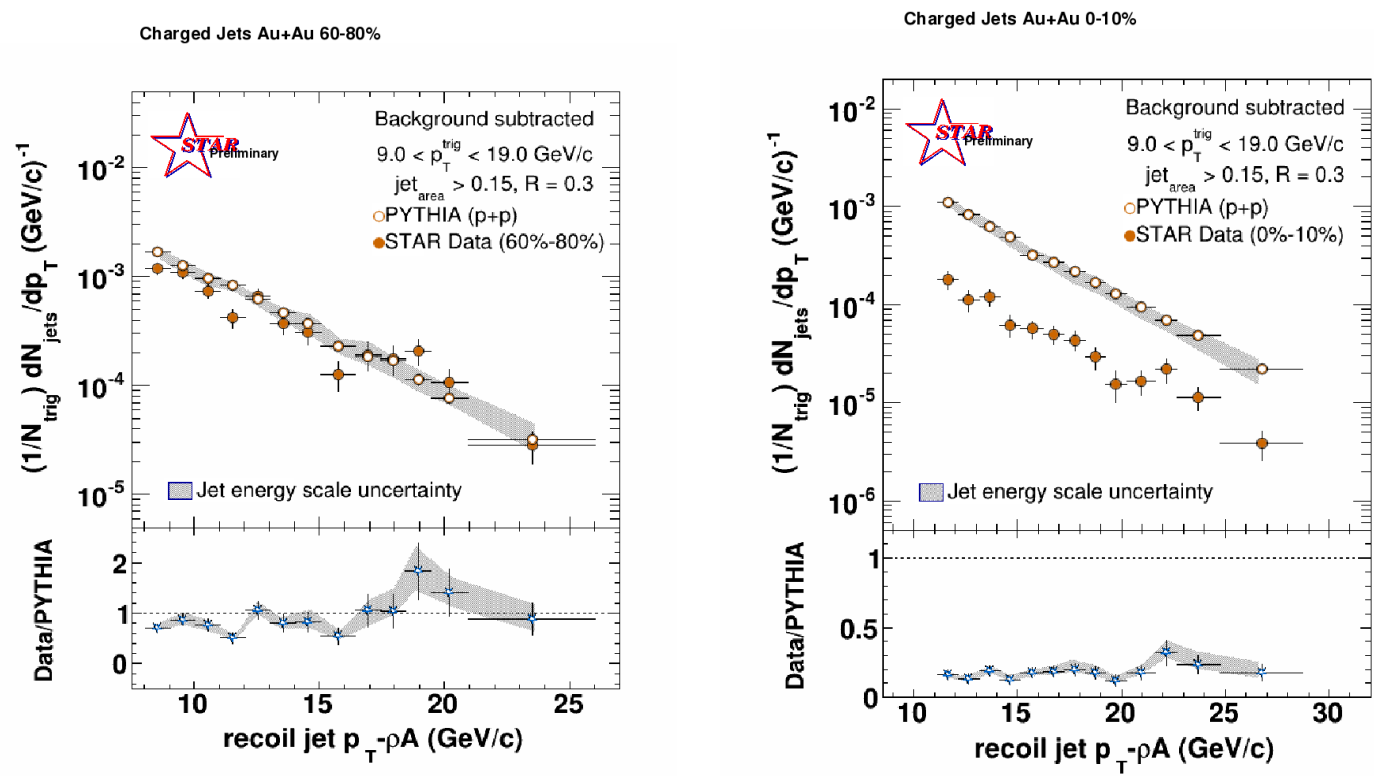

Figure 5: Charged recoil jet spectrum after background subtracted in peripheral (left) and central (right) $\mathrm{Au}+\mathrm{Au}$ collisions at $\sqrt{s_{N N}}=200 \mathrm{GeV}$ compared with PYTHIA charged recoil jet spectrum smeared with background fluctuations and simulated detector effects.

with $p_{\mathrm{T}, 1}$ and $p_{\mathrm{T}, 2}$ being the transverse momentum of the leading (most energetic) and sub-leading (second most energetic) jet. In the absence of a medium in the elementary collisions, produced pairs of jets have the same $p_{\mathrm{T}}$ and are back-to-back with an azimuthal angle difference $\Delta \phi=\pi$ (at leading order pQCD). In heavy-ion collisions on the other hand it is expected that the presence of the hot, dense and strongly interacting medium would lead to the observation of highly unbalanced di-jets.

In order to study the effect of the fragmentation bias, two jet samples were compared. A highly biased jet sample was obtained by requiring the jet constituents' momentum to satisfy $p_{\mathrm{T}, \mathrm{const}}>2 \mathrm{GeV} / c$. The jet imbalance $A_{\mathrm{J}}^{\text {biased }}$ was then calculated for leading jets with momentum $p_{\mathrm{T}, \mathrm{jet}}^{\mathrm{Lead}}>20 \mathrm{GeV} / c(16 \mathrm{GeV} / c$ for $R=0.2)$ and sub-leading jets with $p_{\mathrm{T}, \mathrm{jet}}^{\text {Subead }}>10 \mathrm{GeV} / c(8 \mathrm{GeV} / c$ for $R=0.2$ ). A second jet sample was created by imposing only minimal cut on jet constituents $p_{\mathrm{T}, \mathrm{const}}>0.2 \mathrm{GeV} / c$. Leading and sub-leading jets were geometrically matched with jets from the first sample. Using this unbiased jet sample the jet imbalance $A_{\mathrm{J}}^{\text {unbiased }}$ was calculated.

As a baseline HT pp events from 2006 embedded into Au+Au MB events from 2007 were also used. These events were created to test whether the jet imbalance is merely due to the fluctuating background under the jets, and not any QGP effect.

Fig. 6 shows calculated values of di-jet imbalance $A_{\mathrm{J}}^{\text {biased }}$ (circles) and $A_{\mathrm{J}}^{\text {unbiased (squares) in }}$ triggered central $\mathrm{Au}+\mathrm{Au}$ collisions (full symbols) and pp events embedded into minimum bias $\mathrm{Au}+\mathrm{Au}$ events (open symbols). Jets with $R=0.2$ (left) possess significant difference between $\mathrm{Au}+\mathrm{Au}$ and pp collisions, both for the biased and unbiased jet sample. However a good agreement between $\mathrm{Au}+\mathrm{Au}$ and pp events in case of $R=0.4$ for unbiased jets (containing soft particles) is observed. Such an energy restoration within the cone of $R=0.4$ has not been observed at the LHC 

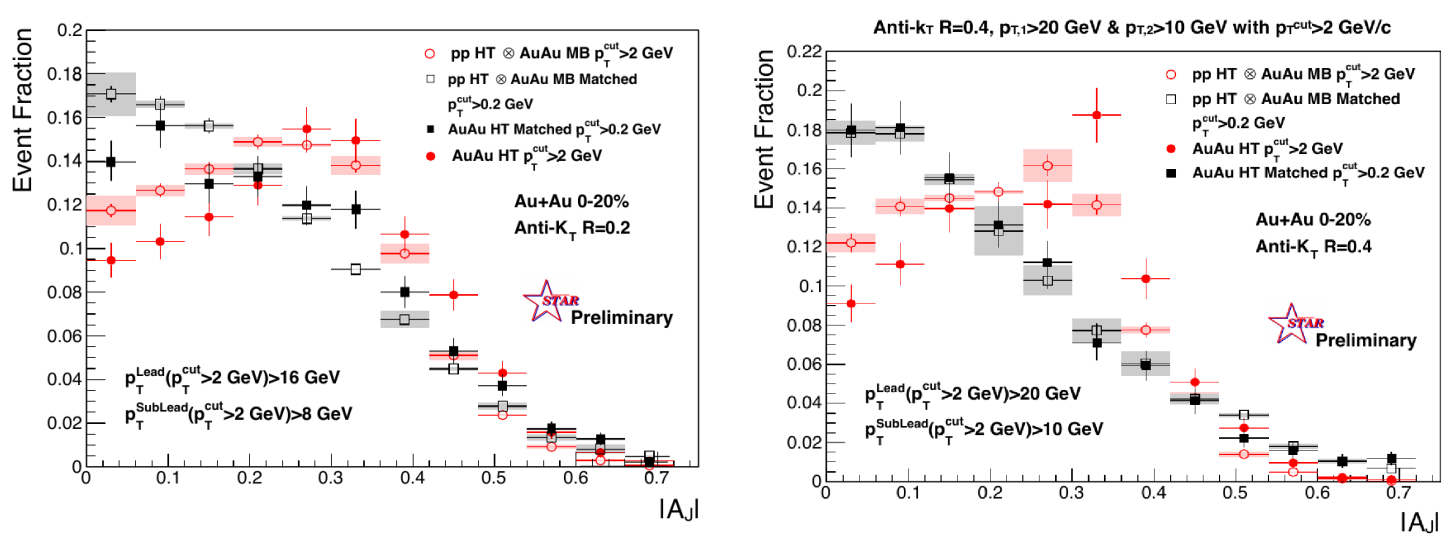

Figure 6: $\left|A_{\mathrm{J}}\right|$ of full jets with resolution parameter $R=0.2$ (left) and $R=0.4$ (right) in Au+Au collisions (full symbols) and pp collisions with embedded $\mathrm{Au}+\mathrm{Au}$ background (open symbols) at $\sqrt{s_{N N}}=200 \mathrm{GeV}$.

$[11,12]$

\section{Conclusions and Outlook}

We have presented recent measurements of inclusive charged jet spectra in central $\mathrm{Au}+\mathrm{Au}$ collisions corrected for the background fluctuations and detector effects. The low momentum part of the presented spectra exhibits a bias due to the cut on the leading hadron momentum. However a valid pp baseline is necessary in order to calculate the charged jet nuclear modification factor $R_{A A}$.

In central $\mathrm{Au}+\mathrm{Au}$ collisions, the semi-inclusive recoil charged-jet spectrum normalized per trigger shows a strong suppression relative to the "detector level" PYTHIA pp spectrum. There is no evidence of suppression in peripheral $\mathrm{Au}+\mathrm{Au}$ collisions.

The measurement of the di-jet momentum imbalance $\left|A_{\mathrm{J}}\right|$ reveals energy recovery in the most central $\mathrm{Au}+\mathrm{Au}$ collisions for larger jets with $\mathrm{R}=0.4$ when low momentum particles are included in the jet. The observation that for $R=0.4$ the energy lost is recovered within the cone of the jet is different than what has been seen at the LHC.

However the $A_{\mathrm{J}}$ measurement and the recoil-jet measurement are significantly different analyses due to a strong selection bias of the $A_{\mathrm{J}}$ measurement (and also some bias due to the hadron trigger in the recoil-jet analysis) and hence their observations should not be directly compared.

All the presented analyses will be further improved by utilizing the high statistics 2014 data with more than one billion $\mathrm{Au}+\mathrm{Au}$ events at $\sqrt{s_{N N}}=200 \mathrm{GeV}$. As a next step, inclusive and semiinclusive jet analyses will also use the information from the BEMC thus extending their kinematic reach.

\section{Acknowledgment}

The work has been supported by the grant 13-20841S of the Czech Science Foundation (GACR).

\section{References}

[1] A. Ali and G. Kramer, The European Physical Journal H 36, 245 (2011). 
[2] M. Gyulassy and M. Plumer, Phys. Lett. B243, 432 (1990).

[3] STAR Collaboration, M. E. Beddo et al., BNL-PUB-5347 (1992).

[4] STAR Collaboration, M. Anderson et al., Nucl. Instrum. Meth. A 499, 659 (2003).

[5] STAR Collaboration, M. Beddo et al., Nucl. Instrum. Meth. A 499, 725 (2003).

[6] M. Cacciari, G. P. Salam, and G. Soyez, JHEP 04, 063 (2008), 0802.1189.

[7] http://www.lpthe.jussieu.fr/ salam/fastjet/.

[8] M. Cacciari, G. P. Salam, and G. Soyez, JHEP 04, 005 (2008), 0802.1188.

[9] STAR Collaboration, P. Jacobs, (2010), 1012.2406.

[10] G. D’Agostini, ArXiv e-prints (2010), 1010.0632.

[11] ATLAS, G. Aad et al., Phys. Rev. Lett. 105, 252303 (2010), 1011.6182.

[12] CMS, D. Krofcheck, PoS ICHEP2012, 418 (2013). 